\title{
Alternative approach to computing transport coefficients: application to conductivity and Hall coefficient of hydrogenated amorphous silicon
}

\author{
Ming-Liang Zhang and D. A. Drabold \\ Department of Physics and Astronomy, Ohio University, Athens, Ohio 45701
}

(Dated: March 30, 2010)

\begin{abstract}
We introduce a theoretical framework for computing transport coefficients for complex materials. As a first example, we resolve long-standing inconsistencies between experiment and theory pertaining to the conductivity and Hall mobility for amorphous silicon and show that the Hall sign anomaly is a consequence of localized states. Next, we compute the AC conductivity of amorphous polyanaline. The formalism is applicable to complex materials involving defects and band-tail states originating from static topological disorder and extended states. The method may be readily integrated with current $a b$ initio methods.
\end{abstract}

The atomistic understanding of the electrical conductivity [1, 2] and Hall coefficient [3 7 are key unsolved problem in the physics of amorphous semiconductors [8]. The challenge is due to two factors: (1) a complex array of localized states of varying physical origin; and (2) at moderate temperature, both localized states and extended states are accessible excited states 9,10 .

Previous work on the conductivity [11, 12] and Hall coefficient [13 17] of amorphous semiconductors has involved either Miller-Abrahams theory or small polaron models [9]. Neither approach is ideal for amorphous semiconductors, with their complex menagerie of localized states due to defects, and tail states due to topological [18] or chemical disorder [8] and electron-phonon couplings strongly dependent upon carrier localization [19].

In this Letter, we first develop a novel formalism for the linear response to a mechanical perturbation 20]. The method properly includes the four possible transitions between extended or localized initial and final states. The full results and many intermediate details are provided in Ref. 21]; here we require only transitions between localized states. Our work resolves the puzzle of the sign anomaly of the Hall mobility in a-Si:H:, provides the temperature dependence of the transport coefficients, and accurately predicts the ac conductivity of polyaniline. The new formalism is expected to be applicable well beyond the examples of this Letter and lends itself to inclusion in current ab initio schemes.

In the small polaron hopping regime, the Kubo linear response formula [20] has been used to compute conductivity and Hall mobility 12, 15 17]. The key mathematical obstacle to computing the current-current correlation function is the imaginary time integral, [12, 15 17] which results from the commutator between the microscopic current and density matrix 20]. For a "mechanical perturbation" (for which an external disturbance may be expressed with additional terms in the Hamiltonian [20]), we can avoid this troublesome commutator, and within this picture the local density operator $\widehat{\rho}$ of a quantity (charge, energy etc.) can be easily constructed 22].

We first average $\widehat{\rho}$ over a state $\Psi^{\prime}(t)$ of the system with the mechanical perturbation, from which the microscopic local density $\rho(\mathbf{r}, t)=\left\langle\Psi^{\prime}(t)|\widehat{\rho}| \Psi^{\prime}(t)\right\rangle$ is obtained. Next we calculate $\partial \rho(\mathbf{r}, t) / \partial t$ by means of the time-dependent Schrodinger equation $i \hbar \partial \Psi^{\prime}(t) / \partial t=H^{\prime}(t) \Psi^{\prime}(t)$, where $H^{\prime}(t)$ is the total Hamiltonian of [system + mechanical perturbation]. The third step is to apply the local density (charge density, energy density) continuity equation: $\partial \rho(\mathbf{r}, t) / \partial t+\nabla \cdot \mathbf{j}_{m}(\mathbf{r}, t)=0 ;$ the microscopic response $\mathbf{j}_{m}$ (current density, energy flux etc.) is then expressed in terms of $\Psi^{\prime}(t)$. Practically speaking, $\Psi^{\prime}(t)$ may be computed to the required order with perturbation theory. By substituting $\Psi^{\prime}(t)$ into the expression for $\mathbf{j}_{m}(\mathbf{r}, t)$, one can obtain the microscopic response to the required order of mechanical disturbance. Spatial and ensemble average are taken at the final stage. The desired transport coefficients can then be extracted from the ensemble average of the spatially averaged flux $\mathbf{j}$.

Since the state of the system is completely determined from the initial conditions, averaging over initial state can be delayed until the final stage. Thus we can avoid the commutator between flux and density matrix, i.e. the integral over imaginary time.

Consider then, a system with $N_{e}$ electrons and $\mathcal{N}$ nuclei in the presence of an electromagnetic field with potentials $(\mathbf{A}, \phi)$, the charge density of state $\Psi^{\prime}$ at $\mathbf{r}$ $\rho^{\prime}(\mathbf{r}, t)=\int d \tau \Psi^{\prime *} \hat{\rho}(\mathbf{r}) \Psi^{\prime}$, where the arguments of wave function $\Psi^{\prime}$ are $\left(\mathbf{r}_{1} \cdots \mathbf{r}_{N_{e}} ; \mathbf{W}_{1} \cdots \mathbf{W}_{\mathcal{N}} ; t\right), \mathbf{W}_{1}$ is the position of the first nucleus, etc. $\widehat{\rho}(\mathbf{r})=\sum_{j} e \delta(\mathbf{r}-$ $\left.\mathbf{r}_{j}\right)-\sum_{L} Z_{L} e \delta\left(\mathbf{r}-\mathbf{W}_{L}\right)$ is the charge density operator. $d \tau=d \mathbf{r}_{1} \cdots d \mathbf{r}_{N_{e}} d \mathbf{W}_{1} \cdots d \mathbf{W}_{\mathcal{N}}$ is the volume element in configuration space. The evolution of the state is determined by the time-dependent Schrodinger equation for which the total Hamiltonian includes the interaction between system and external field. The contribution from the electrons is:

$$
\begin{aligned}
\mathbf{j}_{m}(\mathbf{r}, t)= & \frac{i \hbar e N_{e}}{2 m} \int d \tau^{\prime}\left(\Psi^{\prime} \nabla_{\mathbf{r}} \Psi^{\prime *}-\Psi^{\prime *} \nabla_{\mathbf{r}} \Psi^{\prime}\right) \\
& -\frac{e^{2} N_{e}}{m} \mathbf{A}(\mathbf{r}, t) \int d \tau^{\prime} \Psi^{\prime *} \Psi^{\prime}
\end{aligned}
$$

where the arguments of $\Psi^{\prime}$ are 
$\left(\mathbf{r}, \mathbf{r}_{2}, \cdots, \mathbf{r}_{N_{e}} ; \mathbf{W}_{1}, \mathbf{W}_{2}, \cdots, \mathbf{W}_{\mathcal{N}} ; t\right), \quad d \tau^{\prime}=$ $d \mathbf{r}_{2} \cdots d \mathbf{r}_{N_{e}} d \mathbf{W}_{1} d \mathbf{W}_{2} \cdots d \mathbf{W}_{N_{n}}$. In Eq.(1), the antisymmetry of $\Psi^{\prime}$ under exchange of particles was used, and the Coulomb gauge $\nabla \cdot \mathbf{A}(\mathbf{r}, t)=0$ was adopted to simplify the expression. $\mathbf{j}_{m}(\mathbf{r}, t)$ is gauge invariant [14, 23]. Without the nuclear coordinates, Eq.(11) is identical to the form used by BCS to compute paramagnetic and diamagnetic currents for superconductors in which Bloch states are not radically modified by the electron-phonon (e-ph) interaction 23]. Eq.(11) is a generalization to arbitrary strength of e-ph interaction, and may be used for the localized carriers in amorphous semiconductors or the polarons in ionic and molecular crystals.

We now apply Eq. (1) to compute the conductivity and Hall mobility of an amorphous semiconductor. The ratio of the second term to the first term is $e A / p$ ( $p$ is electron momentum), so that the contribution from the 2nd term may be neglected. Since the carrier concentration is low in lightly-doped amorphous semiconductors, one can invoke the single-electron approximation, and $\Psi^{\prime}(t)$ for $\mathbf{j}_{m}$ may be replaced by the single-electron wave function $\psi^{\prime}\left(\mathbf{r} ; x_{1}, x_{2}, \cdots, x_{3 \mathcal{N}} ; t\right)$, where $\mathbf{r}$ is the coordinate of the carrier, $x_{1}, x_{2}, \cdots, x_{3 \mathcal{N}}$ are the displacements of $3 \mathcal{N}$ vibrational degrees of freedom.

Using perturbation theory, one can expand $\psi^{\prime}(t)$ to the required order of external field [10, 21]. We use A with subscripts to label localized states, denote the coupling between two localized states $\phi_{A}$ and $\phi_{A_{1}}$ caused by external field as $J_{A_{1} A}^{f i e l d}=\int d \mathbf{r} \phi_{A_{1}}^{*} h_{f m} \phi_{A}$, where $h_{f m}=$ $(i \hbar e / m) \mathbf{A}(\mathbf{r}) \cdot \nabla_{\mathbf{r}}+e^{2} \mathbf{A}^{2}(\mathbf{r}) /(2 m)+e \phi(\mathbf{r})$. The spatially averaged microscopic current density to second order of $J^{\text {field }}$ is

$$
\begin{gathered}
\mathbf{j}(\mathbf{s}, t)=-\frac{N_{e} \hbar e}{m \Omega_{\mathbf{s}}} \int_{\Omega_{\mathbf{s}}} d \mathbf{r} \int\left[\prod_{j=1}^{3 \mathcal{N}} d x_{j}\right] \\
\left\{\operatorname{Im}\left(\psi^{(0)} \nabla_{\mathbf{r}} \psi^{(1) *}-\psi^{(1) *} \nabla_{\mathbf{r}} \psi^{(0)}\right)\right. \\
\left.+\operatorname{Im}\left(\psi^{(0)} \nabla \psi^{(2) *}-\psi^{(2) *} \nabla \psi^{(0)}+\psi^{(1)} \nabla \psi^{(1) *}\right)\right\},
\end{gathered}
$$

where $\psi^{(1)}$ is change in state to order $J^{\text {field }}$, and $\psi^{(2)}$ is change in state to order $\left[J^{\text {field }}\right]^{2}$, where $\psi^{(0)}(t)$ is the state of carrier at time $t$ without external field. $\Omega_{\mathrm{s}}$ is the "physical infinitesimal" volume of Kubo 24]. Because the initial state of the phonon-dressed carrier is unknown, we need to average $j_{k}(k=x, y, z)$ over initial phonon distribution and single-electron states.

To compute the conductivity, we only require the order $J^{\text {field }}$ term of Eq.(2). If one applies a voltage drop across the material, the potentials are $\mathbf{A}=0$ and $\phi=-2 \mathbf{E}_{0}$. $\mathbf{r} \cos \omega t$. Using perturbation theory, one can compute $\psi^{(0)}$ and $\psi^{(1)}$ to order $J^{1}$. Substitute $\psi^{(0)}$ and $\psi^{(1)}$ into the first term of Eq.(2), the conductivity from the LL transition is 21]:

$$
\begin{gathered}
\left.\begin{array}{r}
\operatorname{Re} \sigma_{\alpha \beta}(\omega) \\
\operatorname{Im} \sigma_{\alpha \beta}(\omega)
\end{array}\right\}=\frac{N_{e} e^{2}}{\Omega_{\mathbf{s}}} \operatorname{Im} i \sum_{A A_{1}}\left[I_{A_{1} A+} \pm I_{A_{1} A-}\right] \\
\times f\left(E_{A}^{0}\right)\left[1-f\left(E_{A_{1}}^{0}\right)\right] v_{A_{1} A}^{k *}\left(E_{A}^{0}-E_{A_{1}}^{0}\right)^{-1}\left(w_{A A_{1}}^{j}-v_{A_{1} A}^{j}\right), \\
+\frac{N_{e} e^{2}}{\Omega_{\mathbf{s}}} \operatorname{Im} \sum_{A A_{1} A_{3}} f\left(E_{A}^{0}\right)\left[1-f\left(E_{A_{1}}^{0}\right)\right]\left[1-f\left(E_{A_{3}}^{0}\right)\right] \\
\times\left(E_{A}^{0}-E_{A_{1}}^{0}\right)^{-1} \hbar^{-1} J_{A_{3} A}\left[I_{A_{3} A_{1} A+} \pm I_{A_{3} A_{1} A-}\right] \\
+\frac{N_{e} e^{2}}{2 \hbar \Omega_{s}} \sum_{A_{2} A_{1} A} \operatorname{Im}\left(w_{A A_{2}}^{\beta}-v_{A_{2} A}^{\beta}\right)\left(E_{A_{1}}^{0}-E_{A_{2}}^{0}\right)^{-1}\left(v_{A_{2} A_{1}}^{\alpha}\right)^{*} J_{A_{1} A}^{*} \\
\left(Q_{A_{1} A_{3}}\right) v_{A_{1} A}^{k *} \\
+\frac{N_{e} e^{2}}{2 \hbar \Omega_{\mathbf{s}}} \sum_{A_{2} A_{1} A} \operatorname{Im}\left(w_{A A_{2}}^{\beta}-v_{A_{2} A}^{\beta}\right) J_{A_{2} A_{1}}^{*}\left(E_{A}^{0}-E_{A_{1}}^{0}\right)^{-1}\left(v_{A_{1} A}^{\alpha}\right)^{*} \\
\left(Q_{2 A_{2} A_{1} A-}\right) f\left(E_{A}^{0}\right)\left[1-f\left(E_{A_{2}}^{0}\right)\right] \\
\left.\sum_{2+} \pm Q_{2 A_{2} A_{1} A-}\right) f\left(E_{A}^{0}\right)\left[1-f\left(E_{A_{2}}^{0}\right)\right]
\end{gathered}
$$

$\operatorname{Re} \sigma_{j k}(\omega)(j, k=x, y, z)$ takes the positive sign and $\operatorname{Im} \sigma_{j k}(\omega)$ takes the negative sign. Unlike previous theories [9], the dc conductivity may be directly extracted from Eq.(3) without a limiting process. Here, $v_{A_{1} A}^{k}=$ $\int d \mathbf{r} \phi_{A_{1}}^{*}\left(p_{k} / m\right) \phi_{A}$ and $w_{A A_{1}}^{j}=\int d \mathbf{r} \phi_{A}\left(p_{j} / m\right) \phi_{A_{1}}^{*}$ are the velocity matrix elements of a carrier. $f\left(E_{A}^{0}\right)$ is the Fermi distribution. $\quad J_{A_{1} A}=\int d \mathbf{r} \phi_{A_{1}}^{*} \sum_{p \notin D_{A}} U\left(r-\mathcal{R}_{p}\right) \phi_{A}$ is transfer integral from $\phi_{A}$ to $\phi_{A_{1}}$. In Eq.(3), the first term is order $J^{0}$ contribution, the second term is order $J^{1}$ contribution. Here, $I_{A_{1} A \pm}(\omega, T)$ has dimension of time (denote its order as $t_{A_{1} A}^{ \pm}$), and reflects the time evolution of $\psi^{\prime}(t)$ in a field-driven 2-site transition, cf. Eq. (C1) in [21]. The characteristic time $t_{A_{1} A}^{ \pm}$may be interpreted as the mean free carrier time. Similarly the two-fold time integrals $I_{A_{3} A_{1} A \pm}, Q_{1 A_{2} A_{1} A \pm}$, and $Q_{1 A_{2} A_{1} A \pm}$, have dimension of [time] ${ }^{2}$, reflecting the time evolution of $\psi^{\prime}(t)$ in a 3 -site transition induced by transfer integral $J$; they have same order of magnitude $\left[t_{A_{3} A_{1} A}^{ \pm}\right]^{2}$, cf. Eqs.(C3,C7C9) in [21]. $\hbar^{-1} J_{A_{3} A}\left[t_{A_{3} A_{1} A}^{ \pm}\right]^{2}$ is the mean free time in a 3 -site process. The interference between $J^{\text {field }}$ and $J$ is displayed between two components (reached through different paths) of the final state 21]. At high temperature $k_{B} T>\hbar \bar{\omega}$ ( $\bar{\omega}$ is first peak in phonon spectrum), the time integrals $I_{s}$ and $Q_{s}$ can be approximately computed 


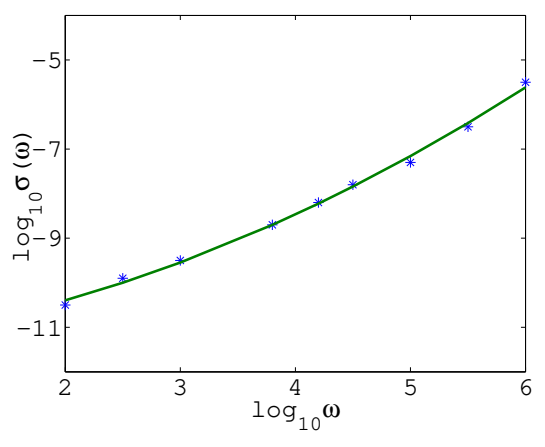

FIG. 1: AC conductivity of polyaniline as a function of frequency at $\mathrm{T}=300 \mathrm{~K}$ : star symbols denote experimental data [25], solid line is from the first three terms in Eq.(4).

using the method of steepest descent (see Appendix D in [21]). For example:

$$
\begin{gathered}
I_{A_{1} A \pm}=e^{-\beta \hbar\left(\omega_{A_{1} A}^{\prime} \mp \omega\right) / 2-\beta \lambda_{A_{1} A} / 4} \\
\left\{C^{-1 / 2} \sum_{n=0}^{\infty} \frac{(-i)^{n}\left(\omega-\omega_{A_{1} A}^{\prime}\right)^{n}}{n ! C^{n / 2}} \Gamma\left(\frac{n+1}{2}\right)\right. \\
\left.-i\left[\sum_{\alpha} \frac{1}{2}\left(\theta_{\alpha}^{A_{1}}-\theta_{\alpha}^{A}\right)^{2} \omega_{\alpha} \cosh \frac{\beta \hbar \omega_{\alpha}}{2}\right]^{-1}\right\},
\end{gathered}
$$

where $\theta_{\alpha}^{A_{1}}$ is the shift in origin of the $\alpha^{\text {th }}$ normal mode caused by a carrier in state $\phi_{A_{1}}, \lambda_{A_{1} A}=\frac{1}{2} \sum_{\alpha} \hbar \omega_{\alpha}\left(\theta_{\alpha}^{A_{1}}-\right.$ $\left.\theta_{\alpha}^{A}\right)^{2}$ and $C=\hbar^{-2} k_{B} T \lambda_{A_{1} A}[10,21]$. The mean free time decreases with increasing $\lambda_{A_{1} A}$ and energy difference: $t_{A_{1} A}^{ \pm} \sim \hbar\left(k_{B} T \lambda_{A_{1} A}\right)^{-1 / 2} e^{-\beta \hbar\left(\omega_{A_{1} A}^{\prime} \mp \omega\right) / 2-\beta \lambda_{A_{1} A} / 4}$. The average mobility $\mu$ is defined by $\sigma_{x x}=$ $e^{2}\left(N_{e} / \Omega_{\mathbf{s}}\right) \mu$. From Eq.(33), one can see that $\mu$ depends on the energy distribution and spatial distribution of localized states. A typical value of $\mu$ can be estimated: $\mu \sim v_{A_{1} A}^{2} t_{A_{1} A}\left(E_{A}^{0}-E_{A_{1}}^{0}\right)^{-1}+$ $v_{A_{1} A} v_{A_{3} A_{1}}\left(t_{A_{3} A_{1} A}^{2} \hbar^{-1} J_{A_{3} A}\right)\left(E_{A}^{0}-E_{A_{1}}^{0}\right)^{-1}$.

As a test, we apply Eqs.(3/4) to the frequency dependence of the ac conductivity in polyaniline at $\mathrm{T}=300 \mathrm{~K}[25]$. The Austin-Mott $\omega^{0.8}$ law 9 does not accurately fit experiments 26]. In Fig 1, we fit the data 25] with the first three terms (a quadratic polynomial of $\omega$ ) in Eq.(4). Because $\omega_{\max }=10^{6} \mathrm{~Hz}<<T=300 \mathrm{~K}$, factors $e^{ \pm \beta \hbar \omega} \approx 1$ do not play a role in the low frequency regime $\hbar \omega<<k_{B} T$.

To describe the Hall effect, one needs $\psi^{\prime}(t)$ to second order of $J^{\text {field }}$ : one $J^{\text {field }}$ includes electric field $E_{x}$, and another includes magnetic field $B_{z}$. After substituting $\psi^{(0)}, \psi^{(1)}$ and $\psi^{(2)}$ into the second term in Eq.(2), and averaging over various initial conditions, $\sigma_{y x}$ is determined from $j_{y}$, see Eq.(49) in [21]. The primary temperature dependence of $\sigma_{y z}$ is included in the time integrals which are obtained from integrating out vibrational states. The 3 -site processes result to two-fold time integrals 21] which have dimension [time $]^{2}$. The time integrals have the same order of magnitude $s_{A A_{2} A_{1}}^{2}$, where $s_{A A_{2} A_{1}}$ is the characteristic time for the 3 -site processes. Similarly the 4 -site processes result to three-fold time integrals [21], the order of magnitude is $s_{A A_{3} A_{2} A_{1}}^{3}$, where $s_{A A_{3} A_{2} A_{1}}$ is the characteristic time for the 3 -site processes. $s_{A A_{2} A_{1}}$ (3-site processes) and $\left[\hbar^{-1} J s_{A A_{3} A_{2} A_{1}}^{3}\right]^{1 / 2}$ (4-site processes) may be explained as the mean free times in presence of magnetic field. Applying fields $E_{y}$ and $B_{z}, \sigma_{x y}$ is inferred from $j_{x}$. The order $J^{1}$ contributions come from various 4 -site processes, their order is $n_{e} e^{2}\left(\hbar^{-1} J_{A_{3} A} s_{A A_{3} A_{2} A_{1}}\right) \hbar^{-2}\left[B_{z} e L_{z}^{A_{2} A_{1}} / m\right] x_{A_{1} A} v_{A_{3} A_{2}}$, where $L_{z}^{A_{2} A_{1}}=\int d \mathbf{r} \phi_{A_{2}}^{*} L_{z} \phi_{A_{1}}$ is the matrix element of the $z$ component of electronic orbital angular momentum. The widely used 3 -site process in the literature $4,7,14-$ [16] is a special case when $A_{1}=A_{3}$. The order $J^{0}$ contributions comes from 3 -site processes. Their order is $n_{e} e^{2} s_{A A_{2} A_{1}}^{2} \hbar^{-2}\left(B_{z} e L_{z} / m\right) x v$. It roughly corresponds to the 'interference' contribution (involving two sites) 16. They are neglected in some other calculations [14, 17]. The ratio of the order $J^{1}$ terms to $J^{0}$ terms is $\left(\hbar^{-1} J_{A_{3} A} s_{A A_{3} A_{2} A_{1}}\right) s_{A A_{2} A_{1}}^{-2} \sim 1$.

Amorphous semiconductors are isotropic, so that one may estimate the Hall mobility as: $\mu_{H}=B_{z}^{-1} \sigma_{x y} / \sigma_{x x} \sim$ $\frac{e}{\hbar} x v s_{A A_{2} A_{1}}^{2} t_{A_{1} A}^{-1}$, where $x_{A_{3} A}=i \hbar v_{A_{3} A}\left(E_{A}^{0}-E_{A_{3}}^{0}\right)^{-1}$. For a-Si:H $[8], \xi$ and $R_{A_{1} A} \sim 5-10 \AA, J \sim 0.02 \mathrm{eV}$, one has $\mu_{H} \sim 0.1-0.2 \mathrm{~cm}^{2} \mathrm{Volt}^{-1} \mathrm{sec}^{-1}$. The temperature dependence of $\mu_{H}$ may be obtained from those of $\sigma_{x y}$ and $\sigma_{x x}$ :

$$
\begin{gathered}
\mu_{H} \sim \frac{e}{\hbar} x v s_{A A_{2} A_{1}}^{2} t_{A_{1} A}^{-1} \\
\exp \left\{-\frac{E_{A_{1} A}^{a}}{2 k_{B} T}-\frac{3}{2} k_{B} T \sum_{\alpha}\left(\hbar \omega_{\alpha}\right)^{-1}\left(\theta_{\alpha}^{A_{1}}-\theta_{\alpha}^{A}\right)^{2}\right\} .
\end{gathered}
$$

At low frequency, the phonon spectral density $\propto \omega^{2}$, so that the sum in Eq.(5) converges. Fig 2 gives the Hall mobility vs. temperature for n-type a-Si:H. Both Eq.(5) and the Friedman-Holstein result 14], are roughly consistent with experimental data [8]. $\quad E_{A_{1} A}^{a}=\lambda_{A_{1} A}(1+$ $\left.\Delta G_{A_{1} A} / \lambda_{A_{1} A}\right)^{2} / 4$ is estimated from typical parameters $\Delta G_{A_{1} A}=0.05 \mathrm{eV}$ and $\lambda_{A_{1} A}=0.2 \mathrm{eV}$ for a-Si [10].

We may also demonstrate the undetermined sign of the Hall voltage from Ehrenfest's theorem. The expected value for the acceleration of a carrier is:

$$
\begin{gathered}
\frac{d}{d t} \int d \tau \psi^{\prime *}(t)\left(\frac{d \mathbf{r}}{d t}\right) \psi^{\prime}(t)=-\frac{q i \hbar}{2 m^{2}} \int d \tau \psi^{\prime *} \psi^{\prime} \nabla \times \mathbf{B} \\
+\frac{1}{m} \int d \tau \psi^{\prime *}(t)\left[q \mathbf{E}-\nabla_{\mathbf{r}} V\left(\mathbf{r},\left\{\mathbf{W}_{\mathbf{n}}\right\}\right)\right] \psi^{\prime}(t) \\
-\frac{q}{m^{2}} \int d \tau \psi^{\prime *} \mathbf{B} \times\left(-i \hbar \nabla_{\mathbf{r}} \psi^{\prime}\right)+\int d \tau \psi^{\prime *} \frac{q^{2}}{m^{2}}(\mathbf{B} \times \mathbf{A}) \psi^{\prime},
\end{gathered}
$$




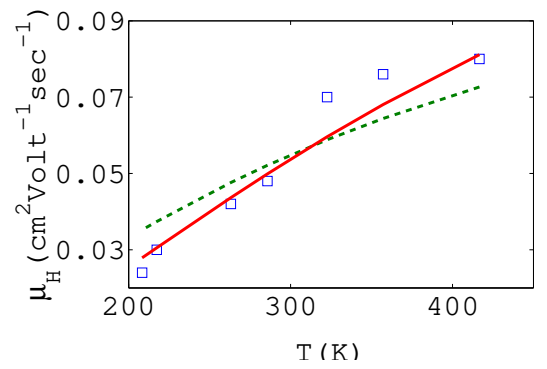

FIG. 2: Hall mobility vs. temperature: square symbols for $\mathrm{n}-$ type a-Si:H[8], dashed line from best fit of Friedman-Holstein formula [14], solid line is from Eq.(5).

where $q$ is the charge of the carrier. If a system has only extended states, because the mean free time of a carrier is much larger than the effective interaction time with phonons and defects, the drift velocity is along the direction of $q \mathbf{E}$ for a nearly free carrier. The direction of the average magnetic force in an extended state (diagonal element) is the same as the classical one $q \mathbf{E} \times \mathbf{B}$, and the sign of the Hall voltage is normal. Because the force exerted on a carrier by the external $\mathbf{E}$ field is much weaker than the binding force of the disorder potential, the time average of $m^{-1} \int \phi_{A_{1}}^{*}\left(-i \hbar \nabla_{\mathbf{r}}-q \mathbf{A}\right) \phi_{A_{1}}$ in a localized states $\phi_{A_{1}}$ is zero: no magnetic force acts on a localized carrier. The mean trapping time that a carrier spends in a localized state before making a transition to other states is [10] $\sim \hbar J^{-2}\left(\lambda k_{B} T\right)^{1 / 2} e^{E_{a} / k_{B} T}$ (high temperature) or $\sim \hbar J^{-2}(\Delta G)$ (low temperature), where $\Delta G$ is the typical energy difference between the final and the initial states, and $\lambda$ is the reorganization energy for a transition. The mean transition time needed for a transition event is $\sim m d R / \hbar$, where $d$ is a typical bond length, $R$ is the distance between two localized states for LL transition, $R$ is localization length for localized-extended transition. The mean transition time is much shorter than the mean trapping time in a typical localized state. Comparing with the transition speed $\hbar /(m d)$, the speed $q E d R / \hbar$ obtained from external electric field $\mathbf{E}$ during the transition time is negligible. The magnetic force suffered by a carrier during a transition is along the direction of $q \mathbf{v} \times \mathbf{B}$, where $\mathbf{v}$ is the transition velocity of the carrier. $\mathbf{v}$ does not have any relation to the direction of $\mathbf{E}$. If one applies $\mathrm{E}$ field along the $\mathrm{x}$ axis and $\mathrm{B}$ field along the $\mathrm{z}$ axis, a direction dependent Hall voltage should be detectable along any direction in the yz plane, not only along the y axis. Checking this prediction would be a critical test for this work. A recent experiment 27] shows that the signs of Hall voltage in several a-Si:H films are not always reverse to those expected from $q \mathbf{E} \times \mathbf{B}$, the present analysis seems to agree with this observation.

We show that localized states are responsible for the anomalous sign of the Hall voltage. The method has the potential to be implemented with current single-particle $a b$ initio simulations, and requires only the eigenvalues and eigenvectors of the single-electron Hamiltonian, dynamical matrix, and quantities easily derived from these.

We thank the Army Research Office for support under MURI W91NF-06-2-0026.

[1] A. Miller and E. Abrahams, Phys. Rev. 120, 745 (1960).

[2] M. Kikuchi, J. Non-Cryst. Sol. 59/60, 25 (1983).

[3] L. Friedman, J. Non-Cryst. Solids 6, 329 (1971).

[4] H. Bottger and V. Bryksin, Phys. Status Solii b80, 569 (1977).

[5] D. Emin, Proc. 7th Int. Conf. on Amorphous and Liquid Semiconductors, ed. W. E. Spear (CICL, Edinburgh) 249, (1977).

[6] L. Friedman and M. Pollak, Phil. Mag. B38, 173 (1978).

[7] B. Movaghar, B. Pohlmann and D. Würtz, J. Phys. C: Solid State Phys. 14, 5127 (1981).

[8] R. A. Street, Hydrogenated Amorphous Silicon, Cambridge Univresity Press, Cambridge (1991).

[9] N. F. Mott and E. A. Davis, Electronic Processes in Noncrystalline Materials, Clarendon Press, Oxford, (1971).

[10] M.-L. Zhang and D. A. Drabold, arXiv:1004.0404, Eur. Phys. J. B. DOI: 10.1140/epjb/e2010-00233-0.

[11] T. Holstein, Ann. Phys. 8, 343 (1959).

[12] I. G. Lang and Yu. A. Firsov, Zh. Eksperim i Teor. Fiz. 43, 1843 (1962) [Soviet Phys.-JETP 16, 1301 (1963)].

[13] T. Holsetin, Phys. Rev. 124, 1329 (1961).

[14] L. Friedman and T. Holstein, Annals of Physics, 21, 494 (1963).

[15] Yu. A. Firsov, Fiz. Tverd. Tela 5, 2149 (1963) [Soviet Phys.-Solid State 5, 1566 (1964)].

[16] J. Schnakenberg, Z. Physik. 185, 123 (1965).

[17] T. Holstein and L. Friedman, Phys. Rev. 165, 1019 (1968).

[18] Y. Pan, F. Inam, M. Zhang and D. A. Drabold, Phys. Rev. Lett. 100206403 (2008); P. A. Fedders, D. A. Drabold and S. Nakhmanson, Phys. Rev. B 5815624 (1998).

[19] R. Atta-Fynn, P. Biswas and D. A. Drabold, Phys. Rev. B 69245204 (2004).

[20] R. Kubo, J. Phys. Soc. Jpn. 12, 570 (1957).

[21] M.-L. Zhang and D. A. Drabold, arXiv:1008.1067, submitted to Phys. Rev. B.

[22] J. H. Irving and J. G. Kirkwood, J. Chem. Phys. 18, 817 (1950).

[23] J. Bardeen, L.N. Cooper and J. R. Schrieffer, Phys. Rev. 108, 1175 (1957).

[24] M.-L. Zhang and D. A. Drabold, Phys. Rev. B81, 085210 (2010).

[25] C. J. Mathai, S. Saravanan, M. R. Anantharaman, S. Venkitachalam and S. Jayalekshmi, J. Phys. D: Appl. Phys. 35, 240 (2002).

[26] A. N. Papathanassiou, J. Phys. D: Appl. Phys. 35, L88L89 (2002).

[27] I. Crupi, S. Mirabella, D. D’Angelo, S. Gibilisco, A. Grasso, S. Di Marco, F. Simone and A. Terrasi, J. Appl. Phys. 107, 043503 (2010). 